\title{
Concept Evaluation of a Reflex Inspired Ball Handling Device for Autonomous Soccer Robots
}

\author{
Harald Altinger ${ }^{2}$, Stefan J. Galler ${ }^{2}$, Stephan Mühlbacher-Karrer ${ }^{1}$, \\ Gerald Steinbauer ${ }^{2}$, Franz Wotawa ${ }^{2}$, and Hubert Zangl ${ }^{1, \star}$ \\ 1 Institute of Electrical Measurement and Measurement Signal Processing, \\ Graz University of Technology, 8010 Graz, Austria \\ 2 Institute for Software Technology, Graz University of Technology, 8010 Graz, \\ Austria \\ team@robocup.tugraz. at \\ http://www.robocup.tugraz.at
}

\begin{abstract}
This paper presents a concept evaluation for a passive ball handling device for autonomous robots that enables the robot to "feel" the ball. A combination of a capacitive and a pressure sensor delivers accurate information of the ball position and movement within the ball handling device- even without touching it. Inspired by the human reflex the sensor values are evaluated to implement a low-level based control loop. This should enable the robot to make minor movement corrections to the overall path calculated by the high-level control system.
\end{abstract}

\section{Introduction}

Playing soccer is not a trivial task. A soccer player has to be fast with good stamina, should be a team-player and should be able to play the ball precisely in the desired direction. This is true for human beings as well as mobile autonomous soccer robots.

Robots do not have any problems with their stamina, but handling the ball is a very difficult task for them, since they do not have a vision and coordination system as humans. For example, an omni-directional vision system of autonomous soccer robots usually has a limited resolution with respect to the localization of the ball in the vicinity of the robot, e.g., an accuracy of about five centimeter. Therefore, a separated ball handling device is important.

Currently, teams in the RoboCup Middle-Size League use two different kinds of ball handling systems: passive and active ones. Active ball handling devices use some kind of actuators to prevent the robot from losing the ball [1]. Passive devices do not use any actuators. Both may use sensors to detect the ball.

We propose a ball handling device that is inspired by humans. We re-build the human reflex system for autonomous robots with high-speed sensors and a micro controller. Instead of manipulating the ball movement we are able to detect and react on the moving ball within milliseconds. Based on the path commanded by the high-level software the micro controller is allowed to adapt the motion

\footnotetext{
* Authors are listed in alphabetical order.
} 
command within limits to prevent the robot from losing control over the ball. The objective of the paper is to evaluate different sensors to be used in the ball handling device.

The paper is structured as follows: Section 2 describes the basic idea of our reflex inspired ball handling device. Following the concept one can find the test setup and the results in Sect. 3. Related work is summarized in Sect. 4.

\section{Concept}

A typical control cycle of a Middle-Size League robot starts with data acquisition using a directed or omni-directional camera system and perhaps some other sensor input transformation. High level software components perform multiple tasks such as self-localization, AI planning, path planning, obstacle avoidance and generate commands for the motion control of the robot hardware. As many high level tasks are involved in this process and huge amounts of data have to be processed, this control loop has a limited reaction time and typically achieves rates of 20 to 50 frames per second.

Inspired by humans we propose a concept of reflexes for mobile autonomous robots. Based on the information obtained by tactile sensors, a underlying loop - a reflex- is introduced. This loop makes minor corrections to the commanded path of the high level system in order to keep the ball within the handling device of the robot. As the complexity of the reflex-loop is much lower than for level control, the reaction time can be significantly reduced.

As an example, if a human soccer player sees a chance to get a ball he or she starts running towards it. This decision includes commands to our muscles to move the legs. In addition, a human has reflexes. When the soccer player steps onto a small stone, reflexes prevent him from falling. reflexes are fast movements not triggered by our brain but some kind of pre-trained actions to be able to react fast on unpredicted situations.

We propose such a high-speed control loop for a ball handling device similar to reflexes for autonomous mobile robots. Figure 1 shows both the high-level cognitive decision loop and the low-level reflex loop. Realizing this device requires

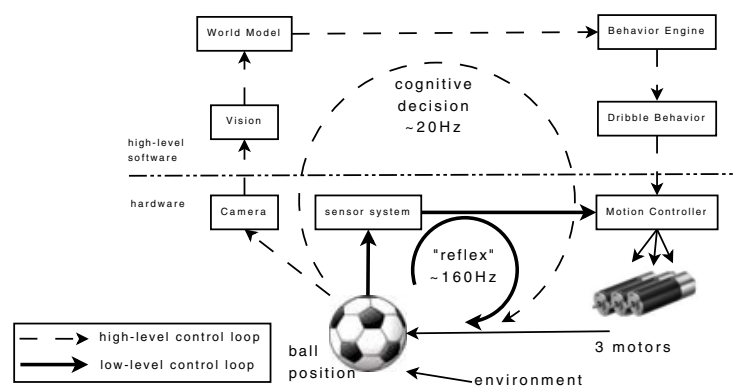

Fig. 1. High-level and low-level control loop 


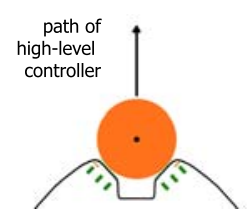

(a)

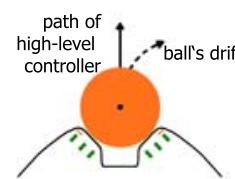

(b)

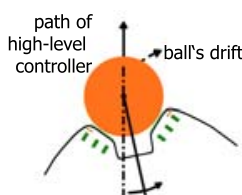

(c)

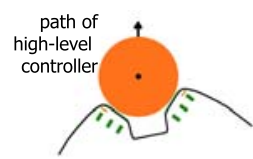

(d)

Fig. 2. Low-level control loop: Within Fig. 2(a) the robot is on the path of the highlevel controller. Figure 2(b) shows a ball trying to drift off which is detected with our sensors mounted on the ball handling device. During Fig. 2(c) the low-level control loop tries to absorb the ball's drift with minor movement corrections. Finally at Fig. 2(d) the robot is back on the path of the high-level controller.

accurate and high-speed sensors that emulate the human capabilities of sensing its environment, and low-level computer power for reacting on unexpected changes in the environment. Implementing this ball handling device allows the robot to calculate minor movement corrections in order to avoid losing the ball.

The following subsections point out two essential components for the proposed low-level reflex-loop. The rest of the paper focuses on the evaluation of the most appropriate sensor technologies for the implementation of this kind of ball handling device.

\subsection{Sensor System}

Comparing the proposed device with human capabilities requires the sensor system to accurately detect the ball position within the ball handling device and nearby the robot. While catching the ball with the device it is also interesting to determine the force a ball applies to the robot. This information in combination with the exact contact point allows the robot to predict the bouncing direction and speed of the ball in an adequate way.

A high sampling rate is the basis for the proposed reflex system. In the RoboCup Middle-Size League robots are moving with up to five meters per second, the ball is kicked with up to eight meters per second. When sampling the RoboCup environment with $100 \mathrm{~Hz}$ the ball may have moved about 13 centimeters between two sensor readings.

\subsection{Measurement Evaluation}

The heart of the reflex based ball handling device is the micro controller reading all sensor data and adapting the motion command of the robot. The high-level software provides information if the robot is currently in dribbling mode and the motion command - consisting of two directional velocity values and a rotational velocity value. If the robot is not in dribbling mode, the motion command is directly passed to the motion controller. The motion controller is responsible for transforming those three velocity values to three actual motor speeds.

Within dribbling mode the reflex mechanism adapts the motion command provided by the high-level software to prevent the robot from losing the ball 
but without completely overwriting the overall moving direction. Basically, we propose to only adapt the robots relative position to the ball to ensure a contact point between the ball an the center of the ball handling device as can be seen in Figure $2(\mathrm{~d})$.

\section{Experiment Setup and Results}

To find the best combination of sensors we experimented with four different sensor types: two contact sensors and two non-contact sensors. The following subsections describe the test setup and the result for each of those sensors in detail.

To compare our results we developed a test setup that allows us to measure all values at the same time. Figure $3(\mathrm{a})$ shows the ball handling device and the location of all evaluated sensors. We distinguish between a static test case and a dynamic test case. Within the static test case we repositioned the ball on a predefined path through the ball handling device. The dynamic test case emulates a real playing situation. The ball is rolled down a ramp to ensure a predefined velocity when it hits the ball handling device (see Figure 4).

A micro controller periodically reads the measurements of the used sensors. The result is sent via controller area network (CAN) protocol to a computer that logs and evaluates the data. Figure 5 shows a general block diagram of the measurement setup.

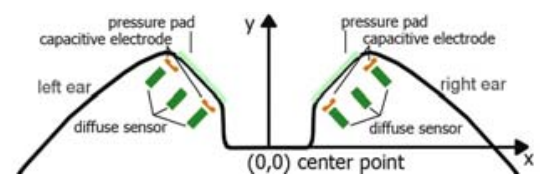

(a) Top view of our ball handling device, which shows the mounting position of three sensors.

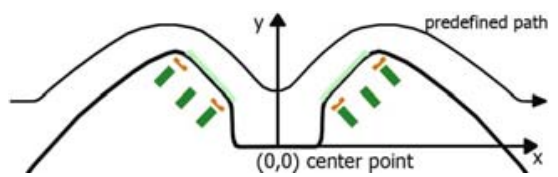

(b) Location of the "center point" and an illustration of the predefined path we use for the static experiments.

Fig. 3. Test setup

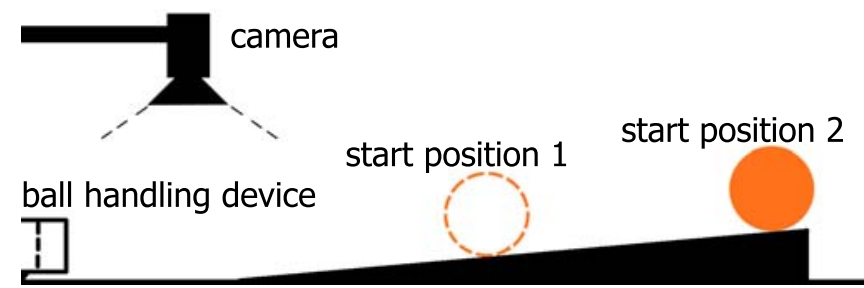

Fig. 4. Setup for dynamic experiments: The ball is placed at 2 different start positions. The first position results in a ball velocity of $0,22 \mathrm{~m} / \mathrm{s}$ when hitting the ball handling device. Start position 2 results in a ball velocity of $0,31 \mathrm{~m} / \mathrm{s}$. The camera is used to determine the velocity. 


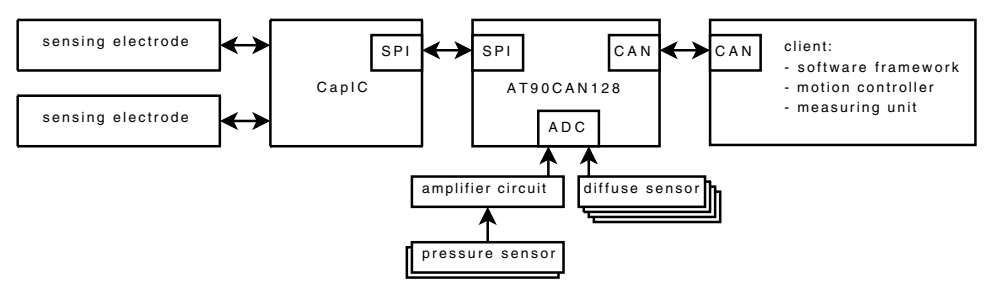

Fig. 5. Block diagram of our measurement setup: The micro controller triggers all sensor readings and sends the pre-processed values (filtering, amplifying, averaging) via CAN to all interested clients. For the test setup the client is a logging and evaluating unit on a computer.

\subsection{Micro Switch}

Humans can precisely detect which parts of their hands have contact with an object. To rebuild this for the robot, multiple micro switches could be used. Therefore, multiple micro switches are mounted on the ball handling device, which allow the robot to detect where the ball hits the device. We use two different arrangement on each side, one with a $6 \times 1$ grid, one with a $3 \times 2$ grid adopted to the contact line between a ball and the ball handling device.

All evaluated commercial micro switches are not able to detect when the ball hits the ball handling device due to the low force a ball applies onto it. Our experiments show a gliding friction of about $1 \mathrm{~N}$ and a rolling friction of about $\frac{1}{10} N$. Therefore, this sensor is not applicable for the reflex based ball handling device.

\subsection{Pressure Sensor}

Similar to micro switches the pressure sensor is a representative of a contact sensor. It consists of a pressure pad filled with air and a silicon piezo resistive absolute pressure sensor SCCP30ASMTP [2] from Honeywell. Applying force onto the pressure pad compresses the air inside which can be detected by the connected sensor. In contrast to micro switches the signal is analog and proportional to the applied force.

Sommer 3 uses pressure sensors to detect collisions of the robot with objects and other robots. In our case we mount two pressure pads on the ball handling device. One on the left and one on the right side of the ball handling device, which enables us to determine on which side the ball touches the robots (see Figure 3(a). Due to little dribbling energy of the ball the signal has to be pre-processed by an amplifier circuit. The amplifier consists of three stages. After the first amplifying phase, the signal is filtered and once more amplified. Corresponding to the amplitude of the analog signal the velocity of the ball hitting the ball handling device can be determined up to $0.25 \mathrm{~m} / \mathrm{s}$, above the amplifier circuit is designed to deliver maximum scale. Due to the characteristic of the amplifier circuit we get a signal even if the ball hits the pressure pads very 

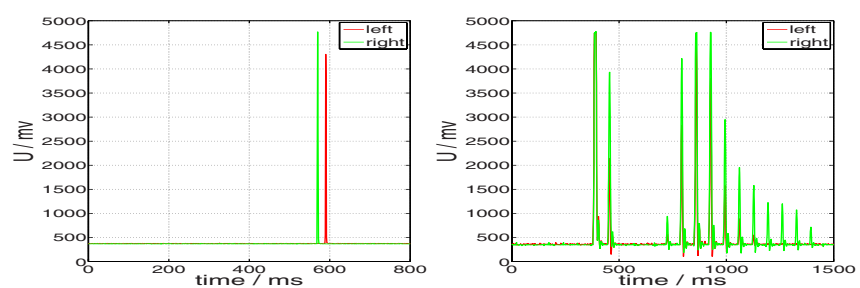

Fig. 6. Left picture, sensor signal from the pressure pad dynamic case: The analog signal range is in-between 0 and $5 \mathrm{~V}$. The ball hits the right short before the left pad, the noise is negligible. Due to this measurement values the ball's position can be distinguished clearly between left and right. Right picture, sensor signal from the pressure pad bouncing: To simulate dribbling we bounced the ball with a cam lever. At the beginning both pressure sensors triggers at the same time which means that the ball's position is in the center of the ball handling device. Between $900 \mathrm{~ms}$ and $1150 \mathrm{~ms}$ it is clearly detectable that the balls position drifts from the center point to the right ear while the applied cam lever force decreases.

smoothly. The micro controller samples this signal with $1 \mathrm{kHz}$. This information can be used to detect if the ball is bouncing.

\subsection{Diffuse Sensor}

Diffuse sensors belong to the non-contact sensor category. We use the OHDK 10P5101 sensor [4] from Baumer Electronics Ltd, which is a diffuse sensors with background suppression and a binary output signal. Either an object is within the pre-defined trigger distance or not. The OHDK 10P5101 can be configured to trigger at distances between three and $130 \mathrm{~mm}$ with an accuracy of $0.2 \mathrm{~mm}$. The sensor readings are provided at $40 \mathrm{~Hz}$. It is not necessary to pre-process the signal of this sensor.

Combining multiple diffuse sensors with different distance configurations allows the robot to locate the ball position. Figure $3(\mathrm{a})$ shows the setup of all six diffuse sensors used in our test setup.

To emulate a similar behavior as the capacitance sensor (see Sect. 3.4) provides we adjust the trigger distances of all six sensors to three different threshold values $(0.5 \mathrm{~cm}, 1.5 \mathrm{~cm}$ and $5.5 \mathrm{~cm})$ on each side of the ball handling device. Due to the fact that those sensors use laser class two diodes as their light source we needed to adjust the height of the sensor mounting to different positions for each side to prevent interferences. Figure 7 visualizes the resulting sensitivity of the test setup.

\subsection{Capacitance}

Capacitive sensors are capable to determine measurands that, in some way, affect the coupling capacitance between two or more sensing electrodes [5]. The simplicity of the sensor elements is unparalleled: They essentially consist of two or more conductive areas. Despite this simplicity, capacitive sensing technology 


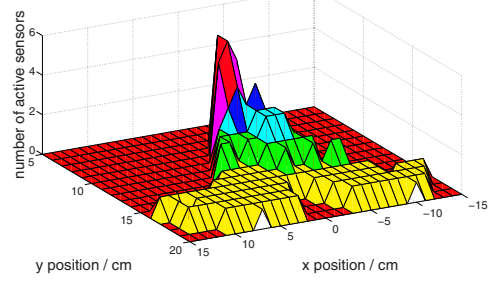

(a)

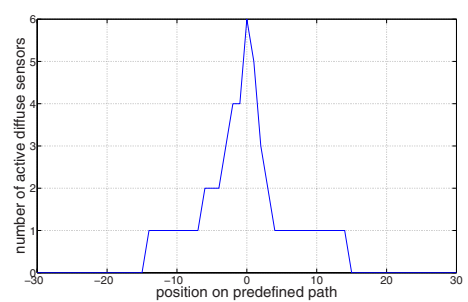

(b)

Fig. 7. Left picture, sensitivity graph of diffuse sensors: With this setup a clear decision can be made if the ball is within the center of the ball handling device or not. The outer sensors realizes a far field detection, the mid and inner sensors a near field. This results within an increasing sensitivity into the direction of the center. Due to the shape of the ball handling device the resulting sensitivity area is asymmetric. Right picture, position on a predefined path (see Figure 3(b) : It shows an increasing number of active diffuse sensors if the ball moves into the center of the ball handling device. Due to the asymmetric ball handling device the curve decreases much more on the right side. This enables us to track the balls movement. The decision between left and right can be made with the knowledge which sensor triggers first.

is very versatile due to a plurality of modes of operation and applicable materials (either conductive or dielectric). With the availability of small and fairly low cost monolithic sensor interfaces (e.g., Cypress CY8C21x34 family, Analog Devices capacitance to digital converters), the acceptance of capacitive technology has constantly increased during the last years.

The circuit used for the reflex based ball handling device is CapIC, a versatile interface IC for capacitive sensing, which was developed at the Institute of Electrical Measurement Signal Processing at Graz University of Technology and Infineon Austria. CapIC is particularly suitable for dynamic observations in various environments due to a high measurement rate and high configurability. The electrodes can be directly exposed to the environment and do not require shielding from external electromagnetic interference. The sensor front-end uses a fully differential approach for an enhancement of the sensitivity as well as the robustness with respect to disturbers and for reduced electromagnetic emissions.

Figure 8 depicts a system model of the sensor interface CapIC. It is based on a carrier frequency principle and uses an array of transmitter electrodes with selectable phase and a differential pair of receiver electrodes. Excitation signals (the carrier) are applied to one (or more) electrodes, the displacement currents injected to a pair of differential receivers are converted to a differential voltage signal by means of shunt impedances, which also form a filter tuned to the carrier frequency. Alternatively, the circuit can also operate in single ended mode, i.e., with only one non-differential receiver. The voltage signal is buffered and amplified in an $\mathrm{HF}$ amplifier stage. Besides amplification, this amplifier is also important to decouple the demodulator from the shunt impedance, as charge injection from the demodulator would lead to undesired resonance effects in the shunt impedances. The HF amplifier is implemented as a linearized 


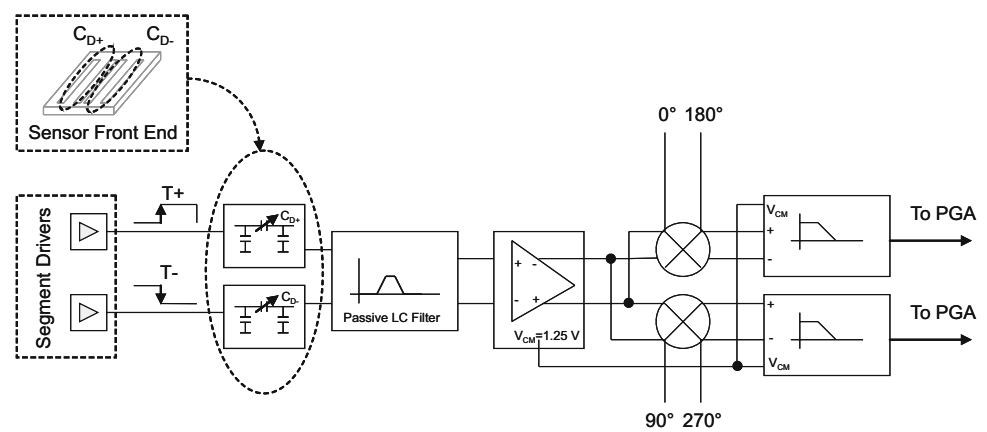

Fig. 8. System model of CapIC [5]: An excitation signal (the carrier) is applied to one (or more) transmitter electrodes, the receivers are connected to passive filters forming a shunt impedance for a current to voltage conversion [6]. The voltage signal is buffered and amplified and subsequently multiplied with the carrier for the I channel and with a $90^{\circ}$ phase shifted carrier and low-pass filtered. The sensor array uses Time Division Multiple Access (TDMA): After one acquisition, other electrode(s) are excited such that all desired capacitances and conductances between the transmitter electrodes and the receiver electrode are obtained after a full sequence of excitation patterns.

differential amplifier stage. The buffered and amplified signal is mixed with the carrier for the I channel and with a $90^{\circ}$ phase shifted carrier for the $\mathrm{Q}$ channel. After the signals are low-pass filtered, offset values due to offset capacitance and offset conductance can be compensated (digitally controllable). Consequently, the gain setting of the Programmable Gain Amplifier (PGA) can be adjusted to make use of the full conversion range of a 12 bit successive approximation $\mathrm{AD}$ converter.

In our test setup we used two pairs of electrodes. One is mounted on the right ear, the other on the left ear of the ball handling device (see figure 3(a)]. Both are connected to the same CapIC circuit which itself is connected to an AT90CAN128 processor board which can be found in [7. The connection between those two components is established via serial peripheral interface (SPI). Based on our CapIC configuration and after averaging we obtain readings at $160 \mathrm{~Hz}$.

The capacitive measurement can be used to determine the ball's position between the left and right side of the ball handling device, however compared with the diffuse sensors it is not possible to ascertain a precise position of the ball based on the current measurement value from one differential pair of electrodes alone. Therefore, a history based signal processing is necessary. Due to the fact that every object (conductive or dielectric) can influence the measured capacitive value the CapIC system can detect a wide variety of objects. Within the RoboCup environment the possible objects are limited to balls, humans and robots which reduces complexity for classification. The ball shows the smallest effect, which causes a short sensing range up to $4 \mathrm{~cm}$. The dead center of the sensing elements can cause wrong measurements. To avoid this, the electrode pairs are mounted $1 \mathrm{~cm}$ behind the surface of the ball handling device. 


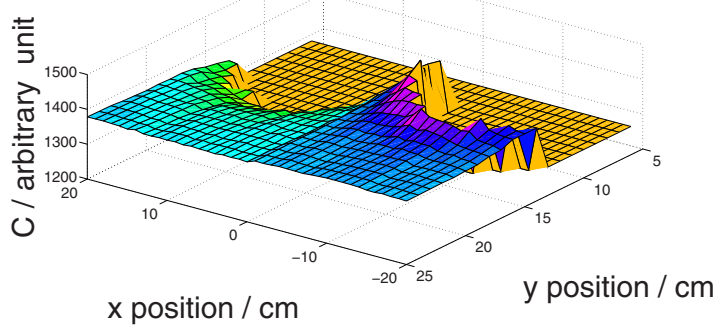

Fig. 9. Sensing area of two electrodes connected to CapIC: The flat area in the back represents the ball handling device. According to the differential measurement the signal within this sensitivity plot shows a valley on the right and a peak on the left side (robots view). The smooth change in the middle is caused by a longtime offset drift from CapIC. Those resulting potential areas are used to localize the ball within the ball handling device.
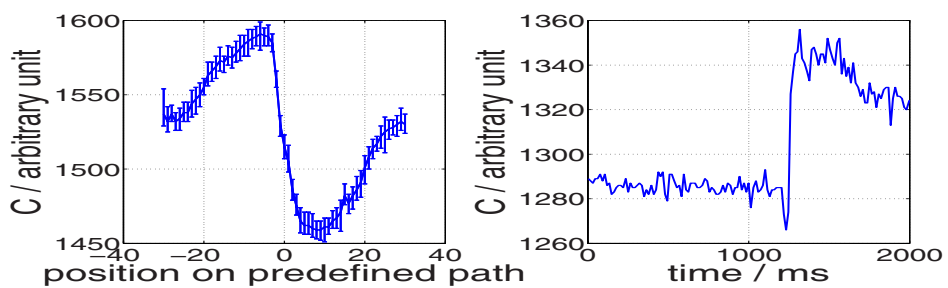

Fig. 10. Left picture, static case: The Ball is repositioned along a predefined path trough the ball handling device. The plotted values represent an averaging the error bars the maximum and minimum before averaging. Combined with the sensitivity plot the first peak indicates that the ball enters the ball handling device on the left side. Second the lines decline which means that the ball goes through the middle and leaves the ball handling device to the right side when the bottom is reached. The starting signal level is the same as the end level which means that the ball is completely out of the sensing area. Right picture, dynamic case: Sensor signal while the ball is rolling down a ramp - to ensure a predefined velocity - straight forward into the ball handling device. It is obvious that the ball hits the right side, bounces on the left and comes to a still stand nearby the right ear of the ball handling device, which can be detect with a higher signal level at the end of the plot compared to the beginning.

\subsection{Comparision of Evaluated Sensors}

Table 1 summarizes all important attributes of the evaluated sensors and the test results. All required values are adjusted to the visions performance. In our test setup the resolution within the $\mathrm{x}-\mathrm{y}$ plane can not be determined for contacting sensors because it is not possible to determine the position of the action point on the pressure pad if the ball hits the ball handling device. Within the rough RoboCup environment it is necessary to protect all sensible components. The 


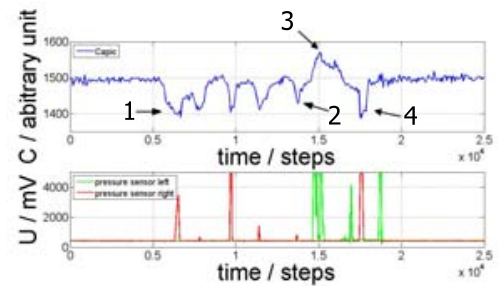

(a)

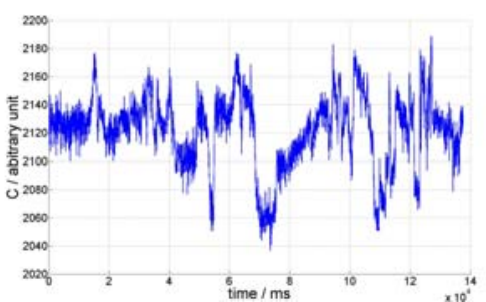

(b)

Fig. 11. Robot is moving on the pitch, trying to dribble the ball: (a) The robots operator can see the robot and the ball. The ball first touches the right side at mark 1 , which can be seen within a decreasing $C a p I C$ value and a short peak from the pressure sensor. The ball starts to bounce on the right hand side of the ball handling device, until mark 2. At mark 3 the ball bounces to the left hand side, which can be seen when the CapIC value increases. Finally the ball is rebound to the right hand side (at mark 4) and leaves the ball handling device. There is a good correspondence between the CapIC measurement an the pressure measurement which can be seen every time the ball touches the ball handling device. (b) Control loop evaluation with a robot operator who neither sees the robot nor the ball. The operator's view is limited to the measurement values displayed on the evaluating computer when making minor movement corrections. An upward trend of the measurement curve indicates the ball is moving to the left side of the ball handling device which requires a movement correction to the left side. A reaction to the right side is required in case of a downward trend. A video analysis shows a good correlation between the measurement curve and the necessary movement corrections to dribble the ball successfully.

Table 1. Comparison of evaluated sensors: The micro switch can not be chosen, see Section 3.1 therefor the pressure sensor is used as contact sensing element. The CapIC sensor satisfy all defined requirements for our ball handling device. In comparison the diffuse sensor has several disadvantages, mainly a low sampling rate, which disqualifies this sensors to be used as the non-contact sensing element.

\begin{tabular}{|c||c|c|c|c|c|c|c|}
\hline & \multicolumn{2}{|c|}{ sampling rate } & \multicolumn{2}{l|}{ resolution x-y plane } & \multicolumn{2}{l|}{ exposed mountable } & costs \\
\hline sensor & required & realized & required & realized & required & realized & calculated \\
\hline & $\mathrm{Hz}$ & $\mathrm{Hz}$ & $\mathrm{cm}$ & $\mathrm{cm}$ & & & $€$ \\
\hline \hline micro switch & $>40$ & $1 \mathrm{k}$ & $<5$ & $\mathbf{X}^{1}$ & $\boldsymbol{V}$ & $\boldsymbol{V}$ & $12 \cdot € 0.2$ \\
\hline pressure & $>40$ & 70 & $<5$ & $\mathbf{X}$ & $\boldsymbol{V}$ & $\boldsymbol{V}$ & $2 \cdot € 25$ \\
\hline diffuse & $>40$ & 40 & $<5$ & 0.02 & $\boldsymbol{V}$ & $\mathbf{X}$ & $6 \cdot € 163$ \\
\hline CapIC & $>40$ & 160 & $<5$ & 1 & $\boldsymbol{V}$ & $\boldsymbol{V}$ & $1 \cdot € 100$ \\
\hline
\end{tabular}

CapIC electrodes and the pressure pads can be mounted on the surface of the robot their electronics can be distributed to protected areas. The diffuse sensors' electronic can not be separated from its sensing elements.

${ }^{1}$ Not determined. 


\section{Related Work}

Typical applications of capacitive sensors are position sensing, material property monitoring such as moisture content or oil quality, proximity switching, occupancy detection, fill level detection etc. Furthermore, capacitive sensing is very common in biomedical and chemical applications. A comprehensive presentation on capacitive sensing can be found in 8 .

The CapIC circuit has been used in a variety of applications and research projects. In 9] a rapid prototyping environment based on CapIC is described. This prototyping environment allows easy configuration of the IC and quick implementation of stand-alone demonstrators. In [6] the circuit has been used for a seat occupancy sensor, with a focuses on the reduction of electromagnetic radiation at higher frequencies. Moisture measurements using the circuit are discussed in 10. The application of the circuitry for personal safety for chain saw users is presented in 11. A more detailed description of the architecture of CapIC and its application to chemical sensing can be found in [5].

Pressure sensors are used in a wide variety of applications such as measurement of fluid/gas flow, liquid level, altitude, chemical process control, and for meteorological observations. Most pressure sensors evaluate a mechanical deformation due to a force associated with the pressure. Examples are strain gauges, piezoelectric and capacitive pressure sensors. However, there are other effects that are also used for pressure sensors, e.g., such as thermal conductivity, ionization and viscosity [12.

Optical sensors are widely used for proximity detection, e.g., in industry for object and material detection, in packaging machines, in robotics, and in laboratory automation. Multiple light sources or detectors are often used to suppress the influence of background light and inclination.

\section{Conclusion and Outlook}

We proposed a reflex based ball handling device that is mainly inspired by humans.

The fact that the high level ball detection with the omni-directional camera only reaches $20 \mathrm{~Hz}$ to $40 \mathrm{~Hz}$, which is too slow for successful dribbling, lead us to a new approach with non-contact and contact sensors. We focused on decreasing reaction time which can be implemented with a low-level control loop. Within this paper we showed an evaluation of four different types of sensors.

However, to get a reliable localization of the ball we decided to use a combination of one contact and one non-contact sensor type. Two pressure sensors to detect which side the ball is hitting within the ball handling device and the CapIC to realize a 'far field' sensing area for localizing the ball. The introduced sensors facilitate a sampling rate at minimum $160 \mathrm{~Hz}$ which is 8 times faster than our current vision based implementation. The CapIC sensor is able to localize the ball within $1 \mathrm{~cm}$ accuracy, which is enough for improved dribbling. All required sensing elements can be mounted at mechanical exposed positions - so the application in RoboCup is possible. Various experiments as described within 
Figure 11(b) show a realizable concept of the reflex loop to be implemented on a micro controller which will be part of future work.

We will concentrate on those experiments and different implementations of the reflex loop and its usability during a RoboCup soccer tournament. Another research focus is on the usability of the capacitance sensor for near-by object detection and classification. Similar to a human who feels when a person comes close to him or her - even without physical contact.

\section{References}

1. Lunenburg, J., v.d. Ven, G.: Tech united team description. In: International RoboCup Symposium 2008, Netherlands, Eindhoven University of Technology (2008)

2. Honeywell: SCC SMT - Absolute pressure sensor (2009)

3. Sommer, U.: Roboter selbst bauen. Franzis (2008)

4. Baumer Electric: OHDK 10P5101 - Diffuse sensors with background suppression (2009)

5. Zangl, H., Bretterklieber, T., Holler, G.: On the design of a fully differential capacitive sensor front-end interfacecircuit. In: Microelectronics Conference ME 2008, Vienna, Austria, October 15-16, pp. 132-136 (2008)

6. Zangl, H., Bretterklieber, T., Werth, T., Hammerschmidt, D.: Seat occupancy detection using capacitive sensing technology. In: SAE World Congress, Detroit, MI, USA, April 14-18 (2008)

7. Krammer, G.: Elektronisches Gesamtkonzept fuer einen mobilen Roboter. Master's thesis, Graz Universtiy of Technology (2007)

8. Baxter, L.: Capacitive Sensors, Design and Applications. IEEE Press, Los Alamitos (1997)

9. Hrach, D., Zangl, H., Fuchs, A., Bretterklieber, T.: A versatile prototyping system for capacitive sensing. Sensors and Transducers Journal 89(3), 117-127 (2008)

10. Fuchs, A., Zangl, H., Holler, G., Brasseur, G.: Design and analysis of a capacitive moisture sensor for municipal solidwaste. Meas. Sci. Technol. 19(2), 9 (2008)

11. George, B., Zangl, H., Bretterklieber, T.: A warning system for chainsaw personal safety based on capacitive sensing. In: IEEE International Conference on Sensors, Leece, Italy, October 26-30, pp. 419-422 (2008)

12. Webster, J.G. (ed.): The Measurement, Instrumentation, and Sensors Handbook. CRC Press LCC, Boca Raton (1999); Springer Verlag, Heidelberg, Germany and IEEE Press 